\title{
Anisotropy of Quasiparticle Lifetimes and the Role of Disorder in Graphite from Ultrafast Time-Resolved Photoemission Spectroscopy
}

\author{
Gunnar Moos, ${ }^{1}$ Cornelius Gahl, ${ }^{2}$ Roman Fasel, ${ }^{3}$ Martin Wolf, ${ }^{2}$ and Tobias Hertel ${ }^{1}$ \\ ${ }^{1}$ Fritz-Haber-Institut der Max-Planck-Gesellschaft, Faradayweg 4-6, 14195 Berlin, Germany \\ ${ }^{2}$ Freie Universität Berlin, Institut für Experimentalphysik, Arnimallee 14, 14195 Berlin, Germany \\ ${ }^{3}$ EMPA Dübendorf, Überlandstrasse 129, 8600 Dübendorf, Switzerland
}

(Received 22 June 2001; published 6 December 2001)

Femtosecond time-resolved photoemission of photoexcited electrons in highly oriented pyrolytic graphite (HOPG) provides strong evidence for anisotropies of quasiparticle (QP) lifetimes. Indicative of such anisotropies is a pronounced anomaly in the energy dependence of QP lifetimes between 1.1 and $1.5 \mathrm{eV}$ - the vicinity of a saddle point in the graphite band structure. This is supported by recent $a b$ initio calculations and a comparison with experiments on defect-enriched HOPG which reveal that disorder, e.g., defects or phonons, increases electron energy relaxation rates.

DOI: $10.1103 /$ PhysRevLett.87.267402

Studies of the ultrafast dynamics of electronic excitations in solids have challenged experiment and theory for years. Advances in ultrafast laser technology and the latest theoretical developments allow us to investigate electron dynamics in growing detail and further our understanding of fundamental scattering processes in solids at the femtosecond time scale $[1,2]$.

For an interacting 3D electron gas the standard theory of $e-e$ scattering - Landau's theory of Fermi liquids - predicts a quadratic dependence of scattering rates on the quasiparticle $(\mathrm{QP})$ energy $\left(E-E_{F}\right)[3]$. In a periodic potential, however, the electronic states are modified with respect to those of a free electron gas and form Bloch states which may result in different QP lifetimes at the same energy if distinct $k$ states within the Brillouin zone are compared. Such anisotropies were indeed predicted by recent $a b$ initio self-energy calculations for $\mathrm{Al}$ and $\mathrm{Be}$ which find strong variations of QP lifetimes for electrons in different bands $[4,5]$. Experimental verification of these predictions, however, remains truly challenging. Time-resolved photoemission from different copper surfaces has revealed some dependence of the measured electron dynamics on the crystallographic surface orientation, but the observed effects could not be clearly attributed to anisotropies of QP lifetimes [6]. Similar experiments on aluminum [7] have likewise not been able to identify band structure effects predicted theoretically [4].

Graphite, a semimetal with layered structure, is expected to be an ideal candidate if band structure effects are to be observed experimentally. The strongly anisotropic band structure of graphite is expected to furnish electron scattering processes with similar anisotropies, leading to anomalous QP lifetimes. The special topology of the graphite band structure has indeed been used to explain apparent deviations of the electron dynamics observed on a cesiated highly oriented pyrolytic graphite (HOPG) surface [8] from the standard predictions for a 3D electron gas [9]. However, the experiments provided no evidence for anomalies that might be associated with anisotropic QP
PACS numbers: 78.47.+p, 71.10.Ca, 79.60.-i, 81.05.Uw

lifetimes. Furthermore, the overlap of the energy range probed experimentally in Ref. [8] and of the range of validity of the approximations used in Ref. [9] is small and calls for more detailed theoretical as well as experimental work.

In this Letter, we present a time-resolved photoemission study of the electron dynamics in HOPG, with particular focus on its energy dependence and the influence of disorder on the relaxation rates. The most striking observation - a pronounced anomaly in the energy dependence of the electron dynamics between 1.1 and $1.5 \mathrm{eV}$ - can be associated with electrons near a saddle point in the graphite band structure at the $M$ point of the Brillouin zone. This and a comparison with experiments on defect-enriched HOPG provide strong evidence for anisotropies of QP lifetimes in graphite which have also been predicted by a recent $a b$ initio calculation [10].

The HOPG sample (Advanced Ceramics) was attached to a tantalum disk which could be temperature controlled from 25 to $1200 \mathrm{~K}$. It was mounted in an UHV chamber with a base pressure of $2 \times 10^{-10}$ mbar. Experiments were performed at room temperature, unless mentioned otherwise. The HOPG sample was cleaved directly before being transferred into UHV and heated repetitively to $900 \mathrm{~K}$. The defect-enriched HOPG surface was produced by Ar-ion sputtering $(0.5 \mathrm{keV}, 7 \mu \mathrm{A}, 300 \mathrm{~s})$. Photoelectron spectra are obtained by means of the time-of-flight technique with an energy resolution of $10 \mathrm{meV}$. For timeresolved pump-probe measurements [11] a visible pump and frequency-doubled probe pulse of typically $85 \mathrm{fs}$ duration are focused on a spot of $50 \mu \mathrm{m}$ diameter on the sample. The photon energy of the probe pulse was chosen to exceed the work function of the sample $(e \Phi=$ $4.50 \pm 0.05 \mathrm{eV}$ ) by typically $0.15 \mathrm{eV}$ in order to probe not only low energy electrons but also hole dynamics.

We start our discussion with a brief overview of processes contributing to the dynamics of photoexcited carriers. After optical excitation the energy absorbed by the electrons is redistributed via electron-electron $(e-e)$ 
scattering, electron-phonon ( $e$-ph) scattering, and transport from the surface into the bulk. In graphite the transport of electrons out of the photoemission detection volume into the bulk is hampered due to the comparatively weak interlayer coupling and is further suppressed by frequent stacking faults in HOPG [12]. Therefore, the dynamics observed in the experiments are expected to be dominated by $e-e$ and $e$-ph scattering processes with $e$-ph scattering becoming increasingly important closer to the Fermi level.

A typical photoelectron spectrum for simultaneous pump and probe excitation is shown in the inset of Fig. 1. The electron dynamics are obtained by plotting the change $\Delta I$ of the photoemission intensity, resulting from excitation by the visible pump pulse, as a function of the pump-probe time delay (see Fig. 1). These cross correlation (XC) traces can clearly be characterized using a biexponential decay with a fast component decaying on the subpicosecond time scale and a slower component decaying on the ps time scale.

A detailed analysis of photoelectron spectra obtained at different pump-probe time delays shows that the electronic system approaches an internal equilibrium with a characteristic time constant of $(250 \pm 50)$ fs. This is estimated using the deviation of photoelectron spectra from the best fit to a Fermi-Dirac distribution [13]. This process - referred to as internal thermalization - is associated with the initial fast decay seen in XC traces and results in an increase in the electron gas temperature by $400 \mathrm{~K}$. This is in reasonably good agreement with the calculated temperature rise using the electronic heat capacity of graphite and the estimated laser power deposited within the optical pen-

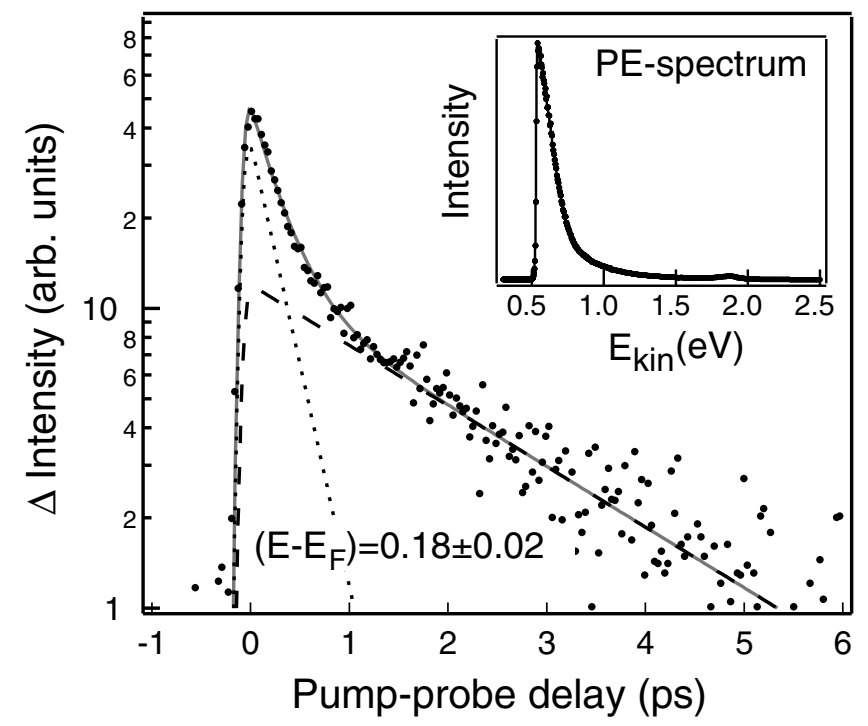

FIG. 1. Cross correlation trace: change of the photoemission intensity in a certain energy interval as a function of the pumpprobe time delay. The pump photon energy was $2.3 \mathrm{eV}$. Fast and slow components of a biexponential fit are indicated by the dotted and dashed lines, respectively. The inset shows a photoelectron spectrum for simultaneous pump-probe excitation. etration depth. The slow component of the $\mathrm{XC}$ traces can be associated with thermalization of the electronic system with the lattice due to $e$-ph interaction $[14,15]$.

In the following we focus on the initial fast decay, associated with the internal thermalization of the electronic system. The corresponding decay rates - as obtained from the fast component of the biexponential fit - are plotted in Fig. 2 as a function of the intermediate state energy $\left(E-E_{F}\right)=E_{\text {kin }}+e \Phi-h \nu_{\text {probe }}$. The data shown in Fig. 2 extend to energies $\sim 0.15 \mathrm{eV}$ below $E_{F}$, and present, to the best of our knowledge, the first 2PPE measurements of electron and hole dynamics within a band crossing the Fermi level.

Obviously the energy dependence of the decay rates cannot be described by a $\left(E-E_{F}\right)^{n}$ dependence with $n=$ 2 as expected for a Fermi liquid nor by $n=1$ as previously predicted for a simplified band structure model for graphite [9]. The most striking deviation from a simple power law is the anomaly between 1.1 and $1.5 \mathrm{eV}$. In this energy range we observe a plateaulike region with no significant increase of the relaxation rates. The error bars in Fig. 2 reflect statistical errors obtained from the biexponential fit after averaging over 13 experimental runs. Systematic errors due to day-to-day variations are somewhat larger.

The most obvious feature in the graphite band structure with which this plateau may be associated is a saddle point of the $\pi^{*}$ bands at the $M$ point of the graphite Brillouin zone [see Fig. 3(a)] and the resulting van Hove singularity at $1.5 \mathrm{eV}$ in the density of states. In contrast to electrons decaying from other regions of the band structure, electrons excited to the vicinity of the $M$ points can decay only by a transfer of comparatively large momentum to

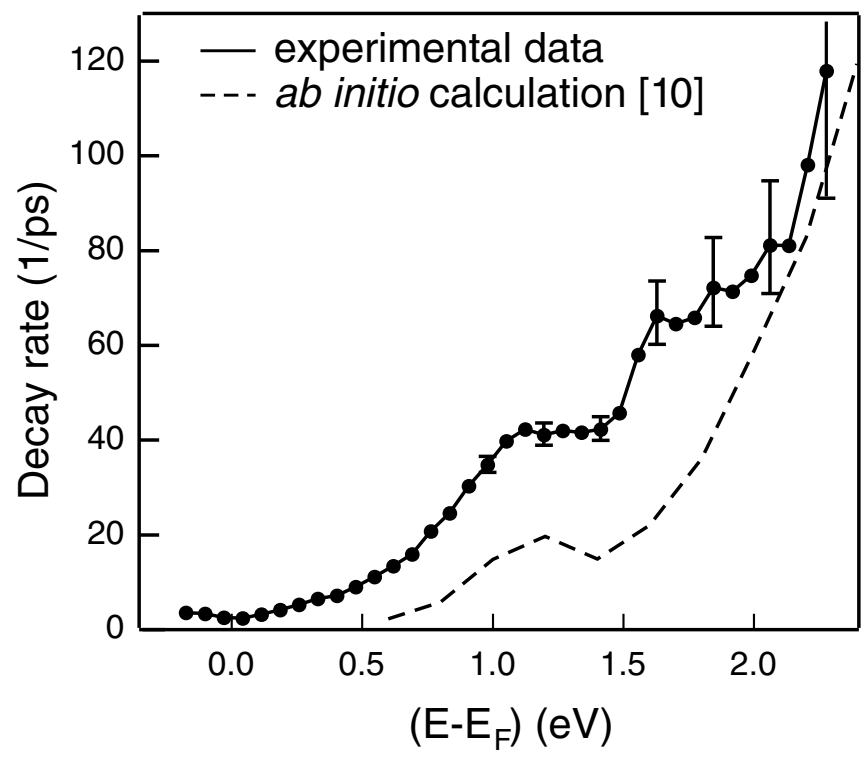

FIG. 2. Energy dependence of decay rates on the pristine HOPG surface. The anomaly near $1.5 \mathrm{eV}$ is indicative of anisotropic QP lifetimes which is also supported by a recent $a b$ inito calculation of QP relaxation rates (dashed line) [10]. 
(a)

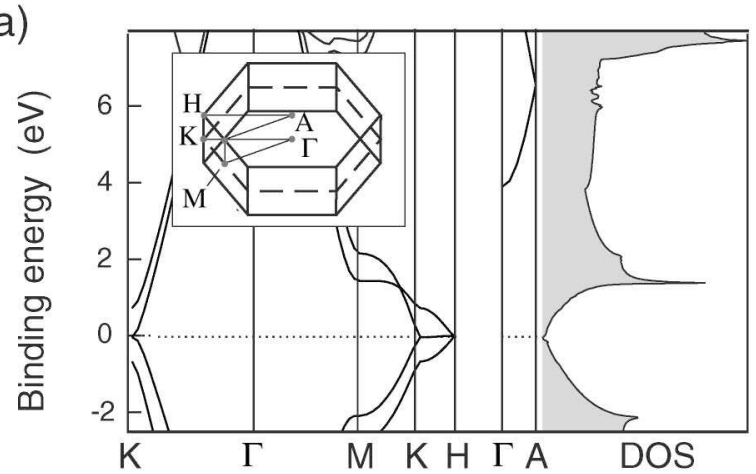

(b)

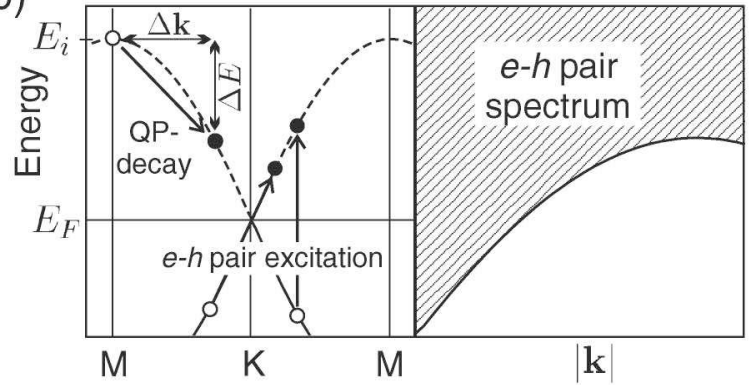

(c)

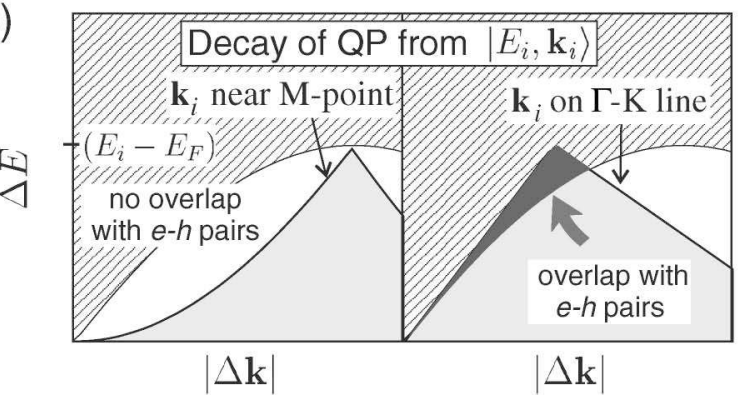

FIG. 3. (a) Graphite band structure along high symmetry directions and density of states. (b) Section of the graphene band structure along the $\overline{M K M}$ line (left panel) and $e-h$ pair spectrum for a graphene sheet (right panel) as well as (c) energy loss versus momentum change for electrons decaying from an intermediate state at the $M$ point (left panel) or an isoenergetic point along the $\overline{\Gamma K}$ line (right panel). The overlap of $e-h$ pair excitation spectra with energy-loss spectra is indicated by the dark grey area.

secondary excitations. Because of the general constraints imposed on scattering processes by energy and momentum conservation, this is expected to result in substantial anisotropies of QP lifetimes in graphite. In particular, one finds that QP excitations in the vicinity of the $M$ points have little overlap with the graphite $e$ - $h$ pair spectrum and are therefore expected to have comparatively long lifetimes. In the following this will be explained in more detail by using a simplified band structure model for a graphene sheet [16].

The qualitative features of QP decay from different initial states can be predicted by comparing the energy-loss spectrum as a function of momentum transfer $|\Delta k|$ for a particular initial state $\left|E_{i}, k_{i}\right\rangle$ with the spectrum of secondary, i.e., $e-h$ pair excitations into which the $\mathrm{QP}$ decays.
The full $e-h$ pair spectrum of graphene is shown in the right panel of Fig. 3(b) (hatched area). Note that due to the special topology of the graphene band structure this is fundamentally different from the parabolic shaped $e-h$ pair spectrum of a free electron gas. Two selected $e-h$ pair excitations on the $\overline{K M}$ line of the graphene band structure are depicted in the left panel of Fig. 3(b), together with a QP decaying from the $M$ point. As stated earlier, the decay of a QP from the $M$ point is associated with comparatively large changes in momentum which lead to the rose-thorn-like shape of the QP energy-loss spectrum [grey area, left panel of Fig. 3(c)]. For such a QP the entire excess energy $\left(E_{i}-E_{F}\right)$ is lost if $|\Delta k|=|\overline{K M}|$ and if the final state is at $E_{F}$ near the $K$ point. Consequently, the overlap of this loss spectrum with the $e$ - $h$ pair spectrum is negligible and decay from the $M$ point via $e$ - $h$ pair generation is improbable due to constraints by energy and momentum conservation. In contrast, the decay of a QP from an isoenergetic point along the $\overline{\Gamma K}$ line, for example, will allow to transfer the excess energy $\left(E_{i}-E_{F}\right)$ if the QP momentum changes by much less. This leads to a significant overlap of the energy-loss spectrum with the $e-h$ pair spectrum [dark grey area, right panel of Fig. 3(c)]. The latter decay process therefore appears to be more probable than decay from one of the $M$ points which would give rise to substantial anisotropies of QP lifetimes in graphene.

We thus speculate that the constraints of momentum and energy conservation also give rise to strongly anisotropic QP lifetimes in graphite and ultimately lead to the anomaly in the experimental decay rates. Note that no comparable anisotropies exist for a free electron gas or for lower energy excitations in graphene. Recent $a b$ initio calculations of QP lifetimes in graphite by Spataru et al. [10] show an anomaly similar to that found in our experiments at about $1.5 \mathrm{eV}$. The authors found pronounced anisotropies in the QP lifetimes with an anomaly arising from electrons in the $\pi^{*}$ band around the $M$ point of the Brillouin zone. If averaged over the entire Brillouin zone-mimicking the averaging process in photoemission due to scattering in the intermediate and final states - the calculated decay rates are reproduced by the dashed line in Fig. 2. The qualitative trends and features of both experimental and theoretical decay rates are evidently the same.

The calculations by Spataru et al. strongly support that the experimentally observed plateau at about $1.5 \mathrm{eV}$ is a direct consequence of anisotropic QP lifetimes in graphite. A quantitative comparison of theoretical and experimental results, however, appears to be difficult. This is most likely because theory does not account for finite temperatures, unavoidable lattice defects, and possible corrections from diffusive or ballistic transport of excited carriers out of the detection volume; effects which are expected to lead to higher decay rates as observed experimentally.

The influence of disorder, e.g., defects or phonons, on the electron dynamics is expected to depend strongly on anisotropies of QP lifetimes. This is best understood if 


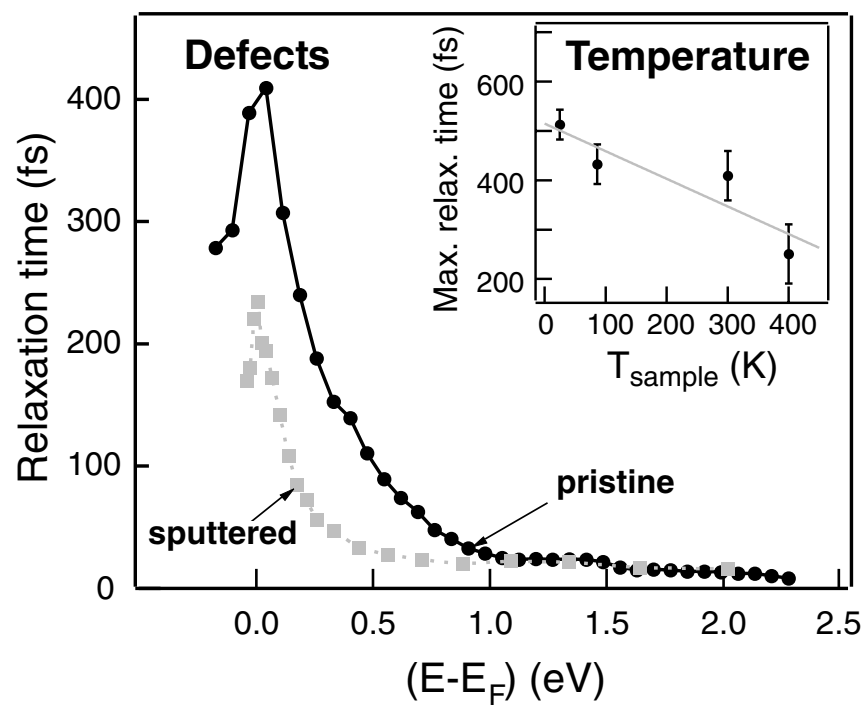

FIG. 4. Influence of different types of disorder on the electron dynamics in graphite. See text.

one considers that lattice distortions break the translational symmetry of the crystal lattice and thereby relax the previously discussed constraints imposed on scattering processes due to the conservation of crystal momentum. As a consequence, both quasielastic scattering between different electronic states as well as inelastic $e$-e collision events will become more frequent and lead to an acceleration of the global electron dynamics.

To test this hypothesis we introduced lattice defects by Ar-ion sputtering. In Fig. 4 we plot the relaxation times of the electron population - the inverse of the rate - as a function of intermediate state energy. Qualitatively we find that the decay of the electronic population is accelerated significantly irrespective of the electron energy.

In agreement with the arguments presented above, these results provide additional evidence for anisotropies in the QP lifetimes in graphite. Furthermore, they extend a recent study on the influence of defects on the electron dynamics in surface states [17] to the dynamics in bulk states.

Alternatively, disorder can also be introduced by heating of the sample, i.e., by an increase of dynamic lattice distortions. Very much like static defects, lattice vibrations and scattering with such can relax the constraints by momentum conservation during QP decay and thereby reduce the measured relaxation times. This is most clearly seen by plotting the decay time found at the peak of relaxation time curves, such as the one in Fig. 4, as a function of lattice temperature (see inset of Fig. 4). We note, however, that this trend may be a superposition of different effects with additional contributions arising from the temperature dependence of $e-e$ scattering rates and enhanced inelastic $e$-ph scattering.

These results may also shed new light on the apparent discrepancies between electron dynamics measured by dif- ferent groups in graphite. Xu et al., for example, observed somewhat shorter relaxation times on a cesiated graphite surface [8] whose absolute values lie in between our results for the pristine and the sputtered surface. Furthermore, as in our experiments on the defect enriched surface, neither Xu et al. nor Ertel et al. [18] found any evidence for a plateau about $1.5 \mathrm{eV}$. This may indicate that surface preparation and defect density plays a more important role in measurements of bulk electron dynamics than previously assumed.

In summary, we have investigated the electron dynamics in HOPG resulting from ultrafast optical excitation. The observed anomaly in the energy dependence of the electron dynamics can be linked to strongly anisotropic lifetimes of electrons excited to about $1.5 \mathrm{eV}$ above the Fermi level. Electrons excited to a saddle point in the graphite band structure at the same energy are expected to be very long lived due to a mismatch of their energy-loss spectrum and the $e$ - $h$ pair excitations available for scattering processes. Disorder is found to accelerate global electron dynamics. This provides further evidence for the anisotropy of QP lifetimes, which has also been predicted by a recent ab initio calculation [10].

We thank W. Ekhardt and A. Rubio for helpful discussions and $\mathrm{M}$. Bovet for kindly providing the graphite band structure. We also thank C. Spataru and his co-workers for sharing theoretical data prior to publication. It is our pleasure to acknowledge G. Ertl for his continuing and generous support. R.F. acknowledges financial support from the Alexander von Humboldt foundation.

[1] H. Petek and S. Ogawa, Prog. Surf. Sci. 56, 239 (1997).

[2] P. M. Echenique et al., Chem. Phys. 251, 1 (2000).

[3] D. Pines and P. Nozieres, The theory of Quantum Liquids, Normal Fermi Liquids Vol. I (Addison-Wesley, Reading, MA, 1989).

[4] W. D. Schöne et al., Phys. Rev. B 60, 8616 (1999).

[5] I. Campillo et al., Phys. Rev. B 61, 13484 (2000).

[6] S. Ogawa et al., Phys. Rev. B 55, 10869 (1997).

[7] M. Bauer et al., Proc. SPIE Int. Soc. Opt. Eng. 3272, 201 (1998).

[8] S. Xu et al., Phys. Rev. Lett. 76, 483 (1996).

[9] J. Gonzalez et al., Phys. Rev. Lett. 77, 3589 (1996).

[10] C. D. Spataru et al., cond-mat/0107043.

[11] E. Knoesel et al., Phys. Rev. B 57, 12812 (1998).

[12] S. Ono, J. Phys. Soc. Jpn. 40, 498 (1976).

[13] T. Hertel and G. Moos, Phys. Rev. Lett. 84, 5002 (2000).

[14] T. Hertel and G. Moos, Chem. Phys. Lett. 320, 359 (2000).

[15] K. Seibert et al., Phys. Rev. B 42, 2842 (1990).

[16] P. R. Wallace, Phys. Rev. 71, 622 (1947).

[17] M. Weinelt et al., Appl. Phys. B 68, 377 (1999).

[18] K. Ertel et al., Appl. Phys. B 68, 439 (1999). 